Boletim Científico do Instituto Agronômico do Estado de S. Paulo

Vol. 31

Campinas, maio de 1972

N. ${ }^{\circ} 16$

\title{
TRATAMENTO DAS SEMENTES COM INSETICIDAS, VISANDO AO CONTROLE DE PRAGAS EM CULTURAS DE ARROZ-DE-SEQUEIRO ( $\left.{ }^{1}\right)$
}

Derly Machado de Souza, engenheito-agrônomo, Seção de Arroz e Cereais de Inverno, Instituto Agronômico, e Clevufas Ramiro, engenheiro-agrônomo, Setor de Desenvolvimento de Produtos e Pesquisas, Companhia Brasileira de Produtos Químicos Shell.

\section{SINOPSE}

Com o intuito de testar a eficiência dos inseticidas Birlane 25\% PM, nas dosagens de 400 e $600 \mathrm{~g} / 60 \mathrm{~kg}$ de sementes, Aldrin $40 \% \mathrm{PM}$, na dosagem de $400 \mathrm{~g} / 60 \mathrm{~kg}$ de sementes, e a mistura de Aldrin 27\% PM + Birlane $25 \% \mathrm{PM}$, na dosagem de $400 \mathrm{~g} / 60 \mathrm{~kg}$ de sementes, aplicados em mistura prévia com as sementes, visando ao controle das pragas de solo em cultura de arroz de sequeiro, foram instalados três experimentos em municípios paulistas e dois em municípios mineiros.

Os esquemas estatísticos utilizados foram o quadrado latino $5 \times 5$ e blocos ao acaso com cinco tratamentos e cinco repetições.

Os resultados mostraram eficiência dos produtos, isolados ou em mistura, nas dosagens empregadas, no controle do cupim e Elasmopalpus, durante o período inicial de desenvolvimento das plantinhas de arroz, que não apresentaram sintomas de fitotoxicidade.

Embora a produção de arroz em casca não tenha mostrado diferença estatística significativa, entre os diferentes tratamentos e a testemunha, notou-se uma tendência de maior produção em todos canteiros tratados.

\section{1 - INTRODUÇÃO}

A cultura de arroz no Estado de São Paulo e em outros estados centrais do Brasil é feita principalmente em terras altas. Sabe-se já, por observações e pesquisas $(\mathbf{1}, \mathbf{7}, \mathbf{8})$, que, nessas condições de cultura, determinados insetos representam fator de relevo na queda

( $\left.{ }^{1}\right)$ Recebido para publicação em 18 de outubro de 1971. 
da produçāo rizícola, embora não se tenha ainda em nosso meio a quantificação dos prejuízos por eles causados.

De muita importância agrícola em certas regiões são os cupins ou térmitas (Cornitermes spp.), insetos da ordem Isóptera (6), família Termitidae, e a lagarta Elasmopalpus lignosellus, Zeller, 1918 - uma Lepidoptera $(\mathbf{1}, \mathbf{7}, \mathbf{8})$, da família Phycitidae.

No combate preventivo a essas duas pragas, tem sido empregado o Aldrin $(\mathbf{3}, \mathbf{4}, \mathbf{9})$, produto recomendado tanto no tratamento de sementes (10) como em polvilhamento do sulco de plantio.

Com o intuito de verificar a possibilidade de substituí-lo ou usá-lo em mistura com outro produto foi realizado este trabalho.

\section{2 - MATERIAL E METODO}

Em 1970, foram instalados cinco experimentos nas regiōes de Morro Agudo, Buritizal, Ituverava (SP), Capinópolis e Ituiutaba (MG), todos em condições de sequeiro.

O delineamento empregado foi o de quadrado latino $5 \times 5$, à exceção daquele instalado em Ituverava, que foi em blocos ao acaso com cinco tratamentos e cinco repetições.

Os canteiros eram constituídos de seis linhas de 5,00 metros espaçadas de $0,60 \mathrm{~m}$, semeadas com uma densidade de sementes de 2 gramas por metro, ou seja, 60 sementes.

Os produtos empregados foram os seguintes:

Aldrin 40\% PM - pó molhável contendo $40 \%$ de $1,2,3,4$, 10, 10-hexacloro-1, 4, 4a, 5, 8, 8a-hexaidro-1, 4-endo, exo 5, 8-dimetanonaftaleno - na dosagem de $400 \mathrm{~g} / 60 \mathrm{~kg}$ de sementes;

Birlane 25\% PM - pó molhável contendo 25\% de 2-cloro-1(2, 4-diclorofenil) vinil dietil fosfato - nas dosagens de 400 e 600 $\mathrm{g} / 60 \mathrm{~kg}$ de sementes;

Aldrin 27\% PM + Birlane 25\% PM na dosagem de 400 $\mathrm{g} / 60 \mathrm{~kg}$ de sementes. tada.

Um dos tratamentos era constituído pela testemunha não tra- 
O tratamento das sementes foi feito na véspera do plantio, colocando-as dentro de uma lata juntamente com o produto, movimentando-se em todos os sentidos por várias vezes.

$\mathrm{O}$ julgamento da eficiência dos tratamentos baseou-se em duas contagens das plantas existentes por tratamento em um metro de cada linha dos canteiros e na produção de arroz em casca das quatro linhas centrais de cada canteiro.

A germinação das sementes de arroz, o desenvolvimento e a produção da cultura foram observados para verificação de possível fitotoxicidade (5) nos diversos tratamentos.

Os experimentos foram mantidos no limpo durante todo o ciclo da cultura.

As variedades estudadas, as datas de plantio, da primeira e da segunda contagens e da colheita constam do quadro 1 .

Quadro 1. - Variedades de arroz e datas dos protocolos referentes a cinco experimentos de tratamento de sementes com inseticidas, realizados em diferentes localidades no ano agrícola 1970-71

\begin{tabular}{l|r|r|r|r|r}
\hline \multirow{2}{*}{ Dados } & \multicolumn{5}{|c}{ Localidades } \\
\cline { 2 - 7 } & $\begin{array}{c}\text { Ituve- } \\
\text { rava }\end{array}$ & $\begin{array}{c}\text { Morro } \\
\text { Agudo }\end{array}$ & Buritizal & Capinópolis & $\begin{array}{c}\text { Ituiu- } \\
\text { taba }\end{array}$ \\
\hline Variedade ........... & $\begin{array}{c}\text { Pratão } \\
\text { Precoce }\end{array}$ & $\begin{array}{c}\text { Bata- } \\
\text { tais }\end{array}$ & IAC-1246 & IAC-1246 & IAC-1246 \\
Data de plantio ..... & $13-11-70$ & $19-11-70$ & $20-11-70$ & $25-11-70$ & $25-11-70$ \\
Data da 1. ${ }^{a}$ contagem. & $27-11-70$ & $12-12-70$ & $11-12-70$ & $9-12-70$ & $9-12-70$ \\
Data da 2. contagem. & $11-12-70$ & $22-12-70$ & $22-12-70$ & $23-12-70$ & $23-12-70$ \\
Data da colheita .... & $6-4-71$ & $15-4-71$ & $5-5-71$ & $12-4-71$ & $16-4-71$ \\
\hline
\end{tabular}

\section{3 - RESULTADOS E DISCUSSÃO}

No quadro 2, onde se encontram os resultados das contagens de plantas existentes, constatou-se que na segunda contagem dos experimentos de Capinópolis e Ituiutaba houve diferença significativa, no número de plantas existentes, entre os canteiros tratados e as testemunhas. Os canteiros tratados não diferiram entre si, em número de plantas. 


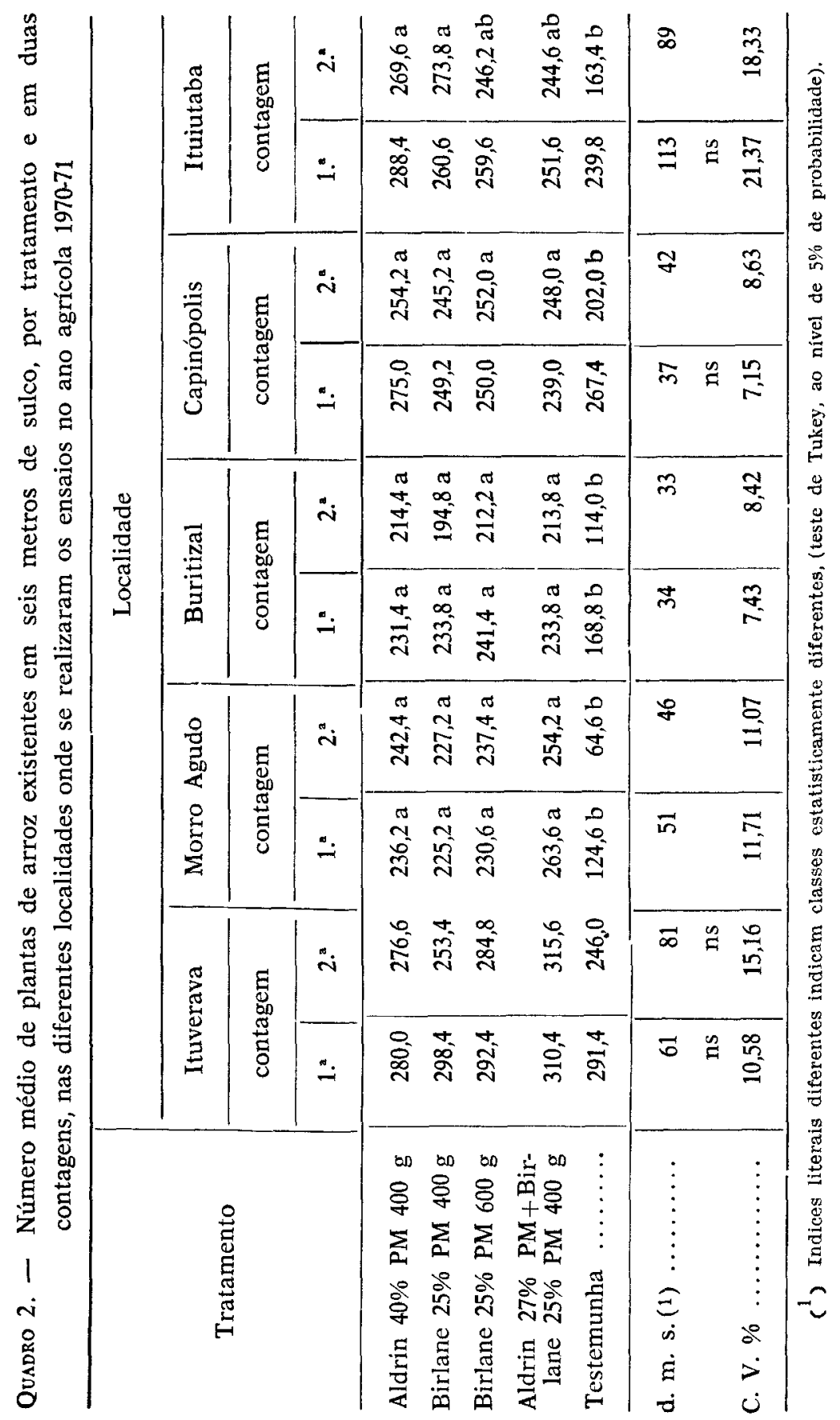


A explicação para esse fato foi que, nesses experimentos, houve um ataque maior de Elasmopalpus, embora fosse constatada a presença de cupins no de Ituiutaba.

Já nos experimentos de Morro Agudo e Buritizal, desde o início de sua instalação os cupins estavam presentes, aparecendo posteriormente Elasmopalpus.

No experimento de Ituverava não se constatou a presença de cupins, apenas de alguns exemplares de Elasmopalpus.

Considerando a produção, não houve diferença estatística entre as médias dos diferentes tratamentos, inclusive as testemunhas.

No entanto, ao serem observados os dados do quadro 3, levando em consideração os números notou-se ligeira tendência de maior produção nos canteiros tratados, quando comparados com as testemunhas.

Quadro 3. - Produções de arroz em casca (gramas por $2 \mathrm{~m}^{2}$ ) obtidas em ensaios de desinfecção de sementes realizados em quatro localidades, no ano agrícola 1970-71

\begin{tabular}{|c|c|c|c|c|}
\hline \multirow{2}{*}{ Tratamento } & \multicolumn{4}{|c|}{ Localidade } \\
\hline & Ituverava & $\begin{array}{l}\text { Morro } \\
\text { Agudo }\end{array}$ & Buritizal & Capinópolis \\
\hline Aldrin $40 \%$ PM $400 \mathrm{~g}$ & 1894 & 2520 & 1510 & 1923 \\
\hline Birlane $25 \%$ PM $400 \mathrm{~g}$ & 1724 & 2070 & 1680 & 1995 \\
\hline Birlane 25\% PM $600 \mathrm{~g}$ & 1862 & 2190 & 1700 & 1893 \\
\hline $\begin{array}{l}\text { Aldrin } 27 \% \text { PM+Bir- } \\
\text { lane } 25 \% \text { PM } 400 \mathrm{~g}\end{array}$ & 1940 & 2420 & 1480 & 1989 \\
\hline Testemunha $\ldots \ldots \ldots$ & 1730 & 1960 & 1350 & 1887 \\
\hline d. m. s. .... & 334 & 836 & 389 & 515 \\
\hline c. V. $\% \quad \ldots \ldots \ldots \ldots$ & 9,40 & 18,59 & 12,51 & 13,18 \\
\hline & ns & ns & ns & ns \\
\hline
\end{tabular}




\section{4 - CONCLUSÕES}

a) - Tanto o Aldrin como o Birlane (nas duas doses) e a mistura dos dois produtos exerceram certo controle sobre as pragas - cupins e Elasmopalpus - durante o período inicial de desenvolvimento das plantinhas de arroz.

b) - Não se notaram sintomas de fitotoxicidade nas plantas de arroz por parte dos inseticidas usados.

c) - Nas condições em que foram realizados os experimentos, não houve diferença de controle entre os produtos usados, significando que poderá ser empregado o Aldrin e o Birlane, separadamente, ou sua mistura, no controle das pragas citadas.

\section{SEED TREATMENT TO CONTROL SOIL INSECTS THAT ATTACK THE GERMINATING RICE SEEDLINGS}

\section{SUMMARY}

Five comparative tests were carried out to compare the efficiency of two insecticides applied to rice seeds prior to sowing to control the attacks of termite (Cornitermes, spp.) and lesser cornstalk borer Elasmopalpus lignosellus (Zeller 1918) on the germinating seedlings. The insecticides tested were: (1) Birlane 25\% WP at the rates of $400 \mathrm{~g}$ and $600 \mathrm{~g}$ per $60 \mathrm{~kg}$ of seeds; (2) Aldrin $40 \%$ WP at the rate of $400 \mathrm{~g}$ per $60 \mathrm{~kg}$ of seeds; and (3) a mixture Aldrin $27 \%$ a. $i$. and Birlane $25 \%$ of equal parts of the two at the rate of $400 \mathrm{~g}$ per $60 \mathrm{~kg}$ of seeds. These four treatments and the control were compared in five different localities (two rice growing areas in Minas Gerais and three in São Paulo). Four tests were planted in $5 \times 5$ Latin aquares and the fifth as a randomized block with five replications.

The average results from the five tests indicate that all treatments were effective in increasing the stand of the plots when compared with the controls. The yields of the treated plots were higher than that of the respective controls, but the differences were not statistically significant.

\section{LITERATURA CITADA}

i. AMARAL, S. \& NAVAJAS, E. Fauna entomológica do arroz e sua importância econômica no Estado de São Paulo. Rev. Agric., Piracicaba 28(3/4):107-124, 1953.

2. BERTELS, A. Combate à bicheira do arroz. Lavoura arroz. 18(210): 16-17, 1964.

3. BESS, $\mathbf{H}$. A. Resistance of soil inseticides for control of subterranean termites. J. econ. Ent. 59:911-915, 1966.

4. BOWLING, C. C. Studies of the effects of Aldrin on seed rice germination and emergence. J. econ. Ent. 57:83-85, 1964. 
5. BOWLING, C. C. Rice water weevil resistance to Aldrin in Texas. J. econ. Ent. 61:1027-1030, 1968.

6. COSTA LIMA, A. M. Isoptera. In: - Insetos do Brasil. Rio de Janeiro, Esc. Nac. Agronomia, 1939. v. 1, p. 263-327. (Série didática 2)

7. ELIAS, R. Pragas do arroz em São Paulo. Bol. Campo 22(218): 3-17, 1967.

8. GIANNOTTI, O.; ORLANDO, A. \& PUZZI, D. Noções fundamentais sobre as pragas da lavoura no Estado de São Paulo e como combatë-las. Biológico 31:231-273, 1965.

9. MARICONI, F. A. M. Inseticidas e seu emprêgo no combate às pragas. 2.ed. São Paulo, Ceres, 1963. p. 144-147, 307-313.

10. MASCARELLO, A. O tratamento das sementes de arroz. Lavoura arroz, 20(232):6-7, 1966. 


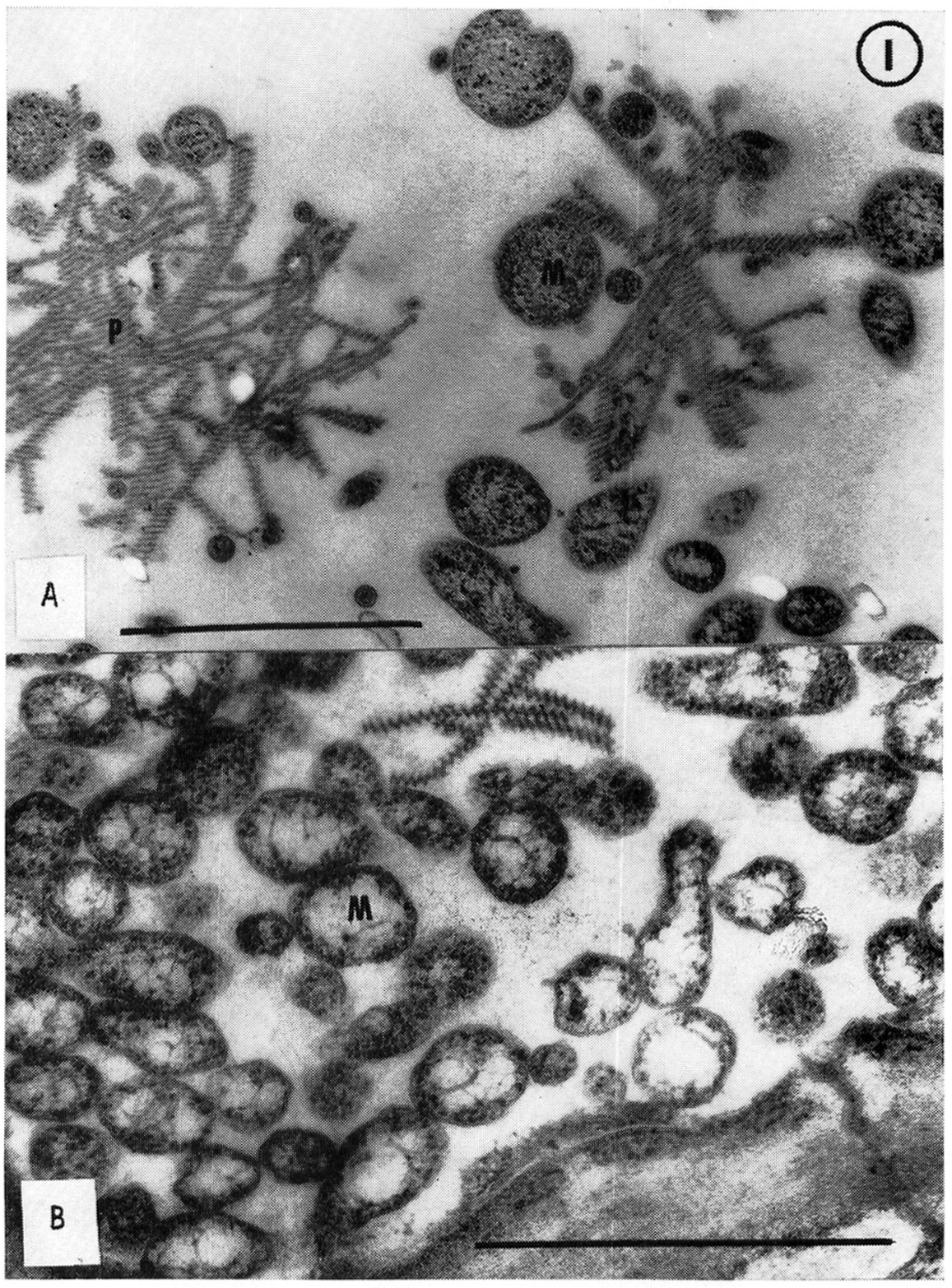

$A$ e $B$. Fotomicrografia electrônica de corpúsculos pleomórficos $(M)$ no interior de vaso crivado de tecido foliar de milho, afetado pela forma vermelha do enfezamento. Além dos corpúsculos notam-se na ilustração partículas alongadas de estrutura helicoidal $(P)$, componentes normais dos vasos crivados de milho. A escala, em todas as micrografias, equivale a $1 \mu \mathrm{m}$. 


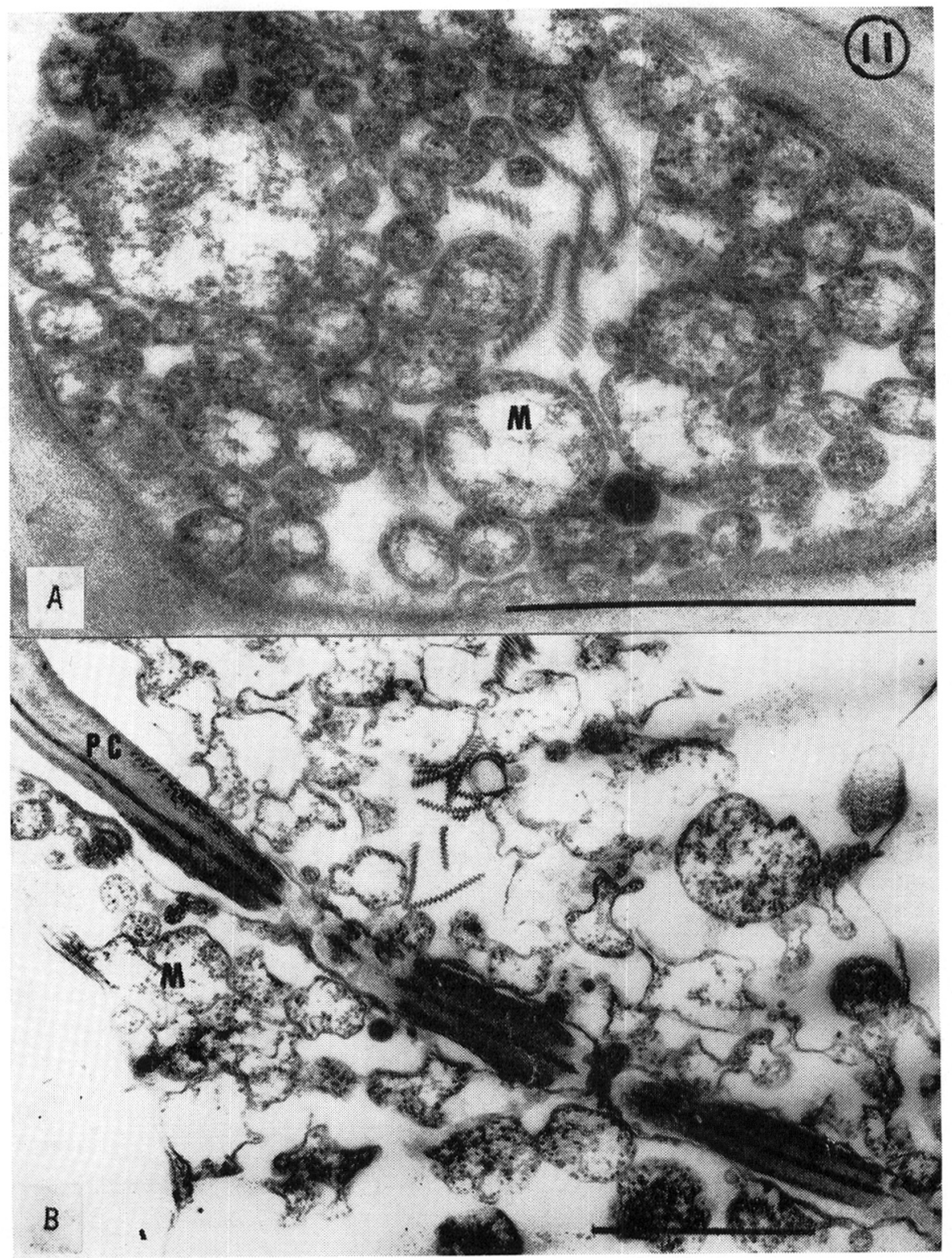

Corpúsculos pleomórficos $(M)$ nos vasos crivados de tecidos foliar $(A)$ e radicular $(B)$ de plantas de milho com sintomas de enfezamento pálido. $P C$ - Placa crivada. 


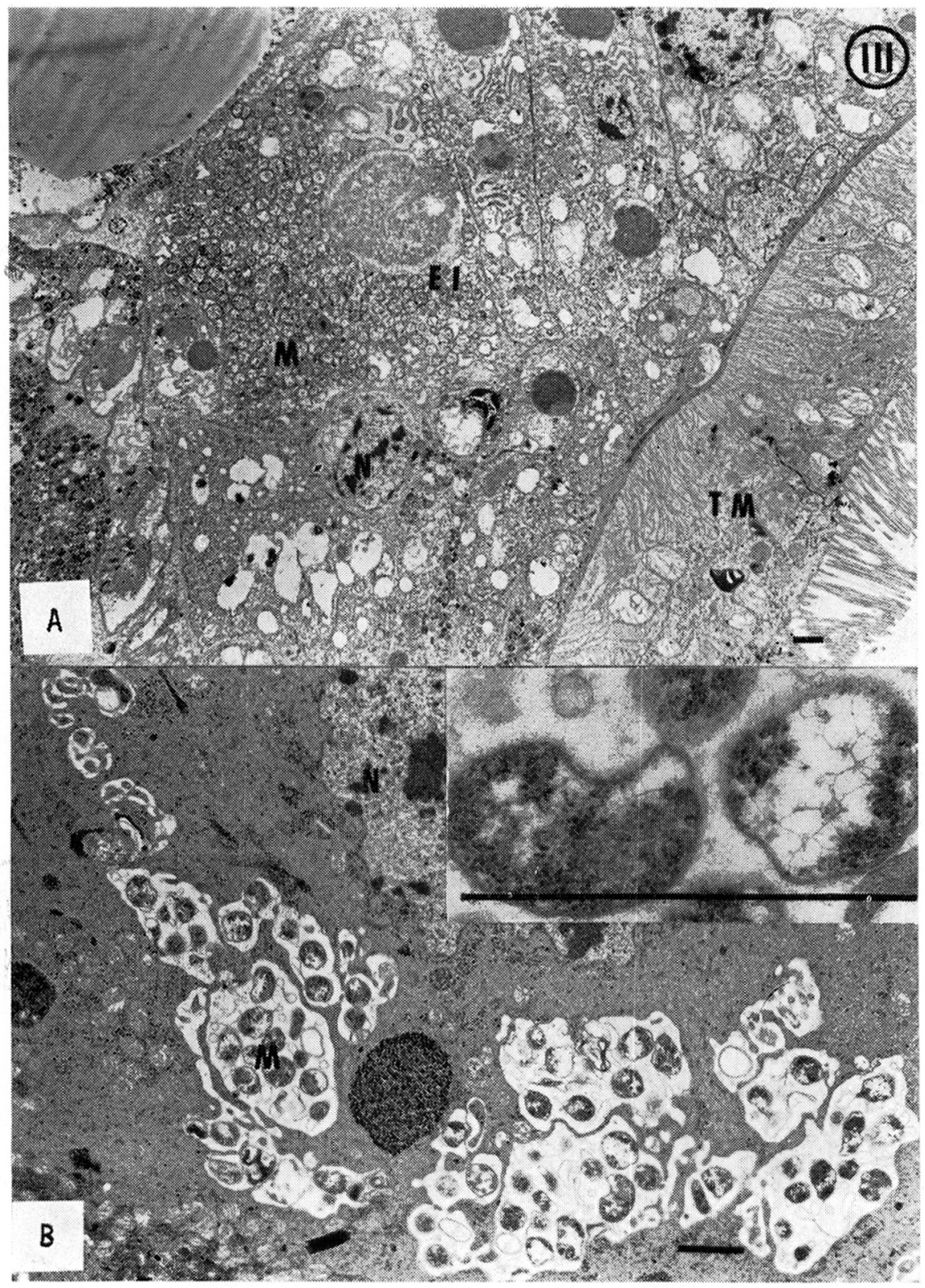

A. Seção ultrafina da região abdominal da cigarrinha Dalbulus maidis, portadora do vírus do enfezamento do milho, forma vermelha. Notam-se células do epitélio intestinal $(E I)$ com número extremamente grande de corpúsculos pleomórficos. A esquerda, parte de um túbulo de Malphigi $(T M)$, aparentemente sem ter sido invadido pelos corpúsculos pleomórficos; $B$ : Parte de glândula salivar de $D$. maidis, com numerosos corpúsculos pleomórficos $(M)$ em cavidades limitadas por membrana. Na inserção, detalhe desses corpúsculos. $N$ - núcleo. 


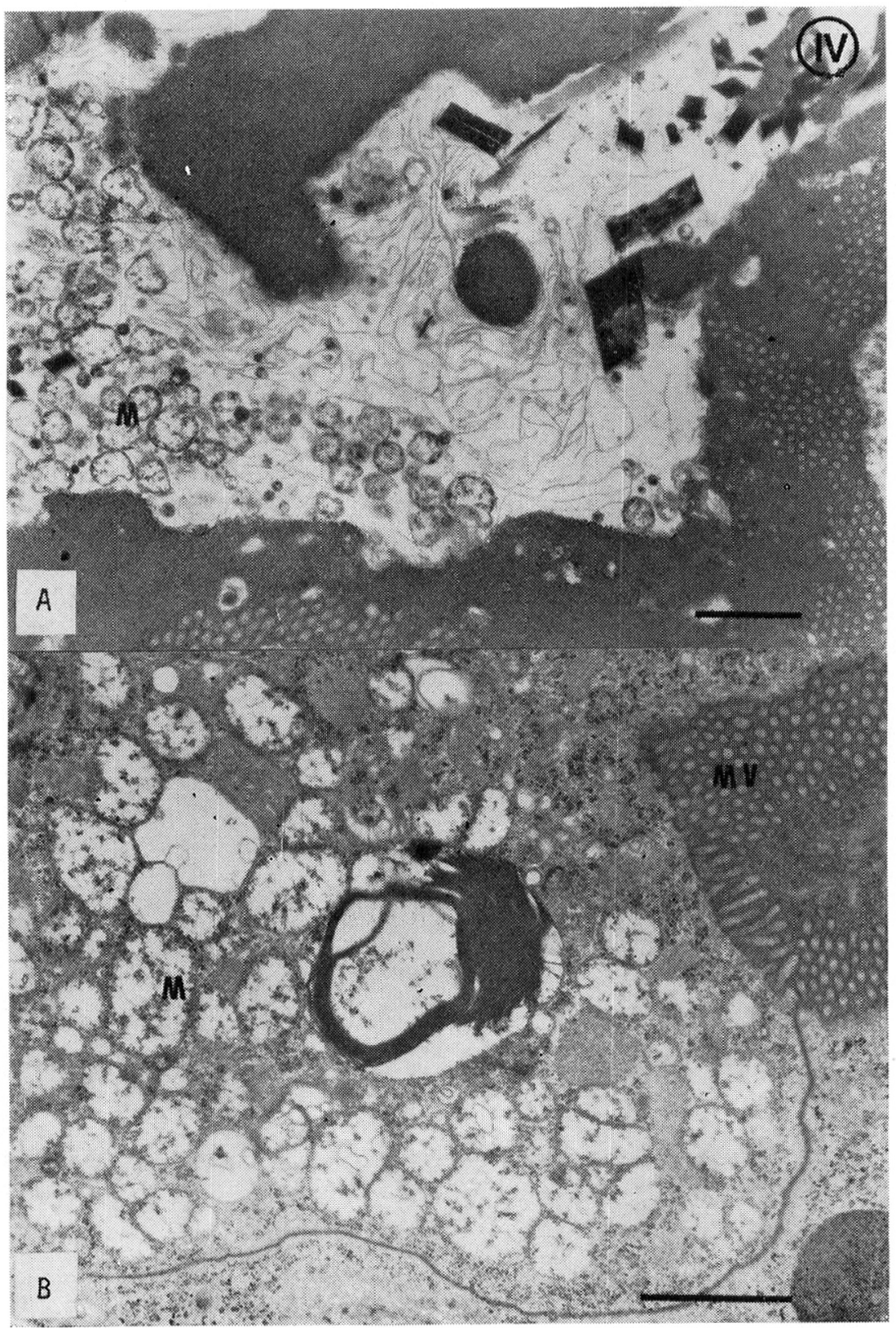

Corpúsculos pleomórficos $(M)$, na luz do intestino $(A)$ e no interior de células epiteliais do intestino da cigarrinha $D$. maidis, portadora da forma pálida do enfezamento do milho. $M V$ - microvilosidade. 


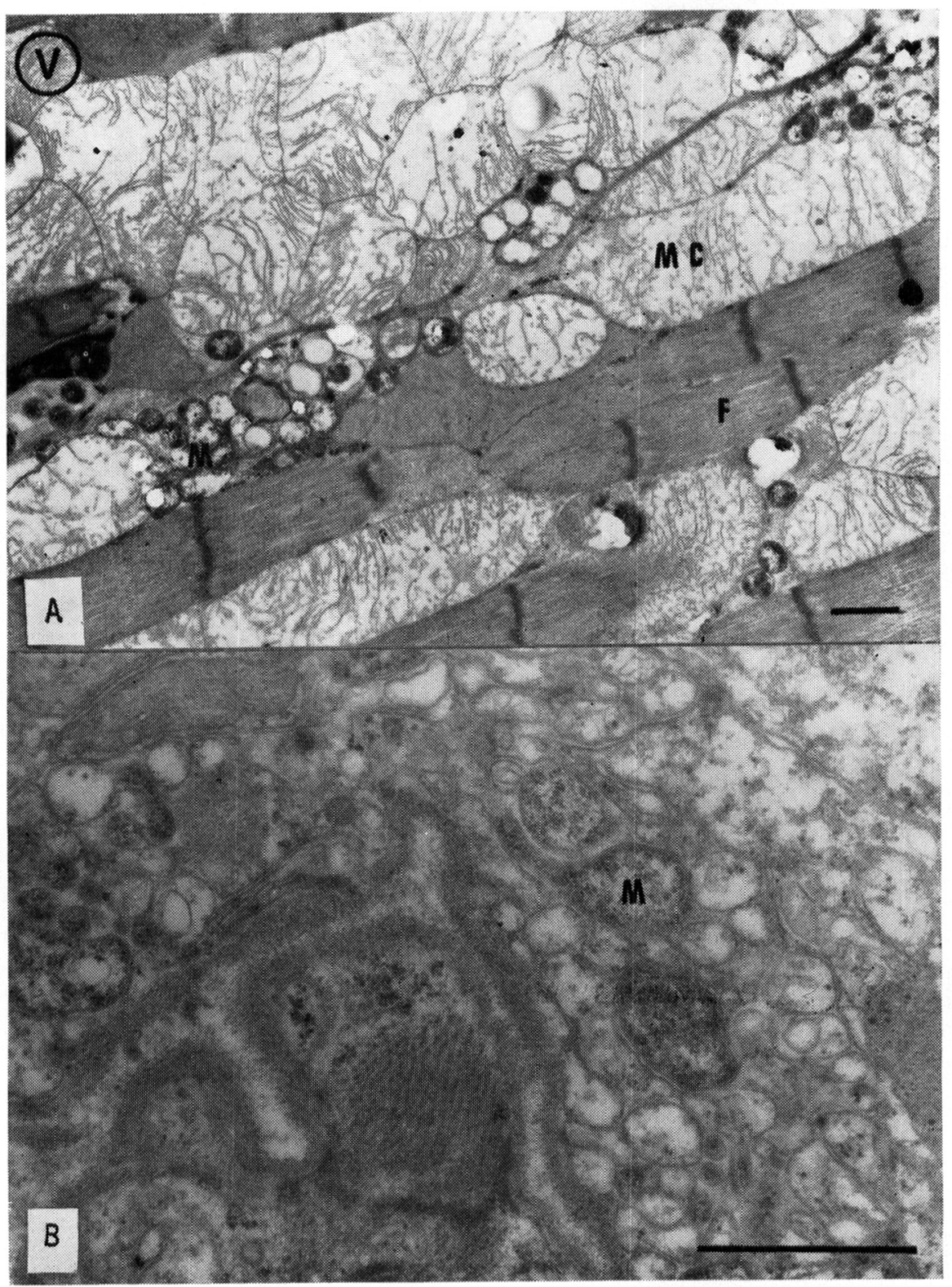

Corpúsculos pleomórficos $(M)$ em células musculares de vôo $(A)$ e na célula epidérmica $(B)$ da cigarrinha. $D$. maidis, portadora do agente do enfezamento do milho, forma pálida. $M C$ - mitocôndrio; $F-$ fibras musculares. 